\title{
Operation of a THTR Ion Source \\ with a Ground Potential Gas Feed into the Neutralizer
}

\author{
J. H. Kamperschroer, L. E. Dudek, L. R. Grisham, R. A. Newman, \\ T. E. O'Connor, T. N. Stevenson, A. von Halle, M. D. Williams, and K. E. Wright \\ Princeton Plasma Physics Laboratory \\ Princeton University, Princeton, NJ 08543
}

\begin{abstract}
TFTR long pulse ion sources have been operated with gas fed only into the neutralizer. Gas for the plasma generator entered through the accelerator rather than directly into the arc chamber. This modification has been proposed for tritium beam operation to locate control electronics at grand potential and to simplify tritium plumbing. Source operation with this configuration and with the nominal gas system which feeds gas into both the ion source and the center of the neutralizer are compared. Comparison is based upon accelerator grid currents, beam comporition, and neutral power delivered to the calorimeter. Charge exchange in the accelerator can be a significant loss mechanism in both systems at high throughput. A suitable operating point with the proposed system was found that requires $30 \%$ less gas than used presently. The extracted $\mathrm{D}^{+}, \mathrm{D}_{2}{ }^{+}$, and $\mathrm{D}_{3}{ }^{+}$fractions of the beam were found to be a function of the gas throughput; at sirailar throughputs, the two gas feed systems produced similar extracted ion fractions. Operation at the proposed gas efficient point results in a small reduction (relative to the old high throughput mode) in the extracted $\mathrm{D}^{+}$fraction of the beam from $77 \%$ to $71 \%$, with concomitant changes in the $\mathrm{D}_{2}{ }^{+}$fraction from $18 \%$ to $26 \%$, and $6 \%$ to $3 \%$ for $\mathrm{D}_{3}{ }^{+}$.
\end{abstract}

\section{MASTER}




\section{INTRODUCTION}

Deuterium-tritium experiments on the Tokamak Fusion Test Reactor (TFTR) are presently planned for 1993-1994. At that time, the fusion power will be comparable to that of the plasma heating power. A likely candidate for the plasma operating mode during these experiments will be the supershot. ${ }^{1}$ Since plasma fuelling in this mode is beam dominated, the use of tritium neutral beams is required to maintain the desired deuterium/tritium ratio in the plasma.

To prepare for these deuterium-tritium experiments, problems associated with running iritium beams in the TFTR neutral beamlines are being assessed. ${ }^{2-5}$ Neutral beam operation up to now, and until deuterium-tritium experiments commence, have been carried out almost exclusively (with the exception of brief periods with hydrogen and neon) with deuterium. Gas conservation efforts with deuterium will be applicable to futurc tritium operation. Geneially, gas for the ion source plasma is injected directly into the plasma generator. Such a system poses a problem for tritium operation since the ion source is at high potential, creating control and feed line isolation difficulties. Ii has been shown that beta decay from tritium gas does nct degrade the ability of the insulated gas feed line to withstand high voltage. 5 Notwithstanding, the problems of control and coping with a tritium leak into the $\mathrm{SF}_{6}$ gas surrounding the ion source remain if tritium is fed to a connection on the plasma generator. This latter problem exists due to the fact that the ion source, which can be operated at potentials up to $120 \mathrm{kV}$, is located in a magnetically shielded enclosure filled to approximately $15 \mathrm{psig}$ of $\mathrm{SF}_{6}$.

These problems are ameliorated and'or eliminated by injecting gas into the grounded neutralizer at a location close to the ion source accelerator. No insulating break in tho gas line is required, the control system is at ground 
potential, and the supply line can be easily double jacketed for tritium containment. Such systems have been proposed for both the Joint European Torus $(\mathrm{JET})^{6}$ and $\mathrm{TFTR}^{3}$ neutral beamlines. While this approach eliminates a high pressure path for tritium leaks into the $\mathrm{SF}_{6}$, the potential for leaks from the ion source into $\mathrm{SF}_{6}$ and of $\mathrm{SF}_{6}$ into the beamline remain and must be dealt with by the tritium recovery system.

To ensure the eflicacy of feeding gas intn the ion source from the neutralizer, several ccncerns must be addressed: is ion source operation affected by the change in gas injection location?; can the beam be adequately neutralized? These concerns are addressed by the experiment reported here.

\section{EXPERIMENTAL APPARATUS}

The relevant components of the beamine for this discussion are the ion source and the neutralizer. Descriptions of the entire TFTR neutral beam injection system $^{7,8}$ and it operation ${ }^{9-11}$ have been published elsewhere. The ion source, ${ }^{12}$ developed by the Lawrence Berkeley Laboratory for the US fusion community, consists of a plasma generator ${ }^{13}$ and an accelerator. ${ }^{1.4}$ Ions created in the plasma generator axe accelerated in a four grid assembly. From high voltage to ground, the four grids of the accelerator are: source grid, gradient grid, suppressor grid, and ground grid. Ions are accelerated in the first two gaps and then decelerated as they pass from the suppressor grid to ground. A deceleration stage is necessary to introduce a negative potential, relative to the beamline ground, to block electrons from the neutralizer plasma from being accelerated into the ion source. 
The neutralizer, $15 \mathrm{~cm}$ by $50 \mathrm{~cm}$ in cross section and $250 \mathrm{~cm}$ long, is closely coupled to the ion source. Gas exiting the ion source must travel the entire length of the neutralizer before reaching the large volume of the beamline and its cryogenic pumps. ${ }^{15}$ This gas, or gas injected directly into the neutralizer, provides a medium with which the beam ions charge exchange to produce a mixed beam of ions and neutrals. A magnet downstream of the neutralizer deflects the ions from the beam path. Beam power is measured on a calorimeter downstream of the magnet.

Initial operation of these ion sources used gas injection directly into the ion source. $^{2}$ Beginning in April 1990, the gas system was modified to provide gas injection into the neutralizer (for all twelve TFTR ion sources) at a location approximately at the neutralizer's midpoint. Gas was then injected simultaneously into both the ion source and the neistralizer. At that time, the gas throughput into the ion source was reduced relative to that of the ion source gas feed only. The net effect, however, was an increase in total gas throughput. Neutralizer gas injection was adopted to ensure that the neutralizer line density was always sufficient to approximate charge exchange equilibrium conditions. One ion source also had an independent neutralizer gas feed added cioser to the ion source (about $50 \mathrm{~cm}$ downstream of the ground grid). Ion source operation and beam parameters are compared for these latter two methods of gas injection. Since the neutralizer gas feed close to the ion source enters the beamline through the Optical Multichannel Analyzer (OMA) diagnostic chamber, ${ }^{16}$ it shall be referred to hereafter as the OMA gas system. When this system was used, the ion source and the central neutralizer systems were disabled. Gas for the plasma generator had to enter the ion source through the accelerator. The central neutralizer gas system will be referred to hereafter as the neutralizer gas system. 
Gas was fed into the ion source via a remotely controlled Veeco PV-10 piezoelectric valve ${ }^{17}$. A solenoid was used to turn the neutralizer gas on and off; a manual needle valve in series with the solenoid was used to control throughput. Due to the manual nature of this valve and its location, throughput into the neutralizer was not varied during the course of the experiment. For OMA gas injection, a remotely controlled Maxtek piezoelectric vaive ${ }^{18}$ was used. Figure 1 is a schematic of these different gas injection locations. Each of the three different feed points mentioned above are indicated. All are fed from a common gas bottle. The ion source together with the PV-10 piezoelectric valve and its local driver electronics are at high voltage. This voltage is stepped down to ground in the three gaps of the accelerator. The neutralizer and its two gas systems are at ground potential.

Beam operation was diagnosed with an OMA which monitors Doppler-shifted light emission from beam neutrals in the neutralizer. ${ }^{16}$ It indirectly measures the relative proportions of the beam extracted as $\mathrm{D}^{+}, \mathrm{D}_{2}{ }^{+}$, and $\mathrm{D}_{3}{ }^{+}$. Factional energy $\mathrm{D}^{\circ}$ created by the dissociation of $\mathrm{D}_{2}{ }^{+}$and $\mathrm{D}_{3}{ }^{+}$are less dəsirable for heating the tokamak plasma than the full-energy $D^{0}$ created from $\mathrm{D}^{+}$. A concern, therefore, is the effect of the gas system on the extracted $\mathrm{D}^{+}, \mathrm{D}_{2}{ }^{+}$, and $\mathrm{D}_{3}{ }^{+}$ fractions.

Waterflow calorimetry was used to measure the neutral beam power on the calorimeter. ${ }^{19}$ If the beam were to become under-neutralized it would be evident as a decrease in power delivered to the calorimeter. Ion dumps, onto which the unwanted ions are deflected, are instrinented with thermocouples. These also provide information on beam neutralization. 
The experiment consisted of operation first with the ion source plus neutralizer gas feed system, and then with the OMA gas system. In the first case, the throughput into the ion source was incrementally reduced, at a fixed neutralizer gas feed of -15 torr- $/$ s, until the ion source became difficult to run. In the second case, OMA throughput was reduced until operational difficulties were again encountered. Difficult operation implies arc faulting or high voltage faulting between grids during beam extraction.

Gas throughput was measured using two techniques. First, the gas valves were calibrated by pulsing gas for one second into the isolated beamline when the cryopanels were at room temperature. The accuracy of this technique is dependent upon the accuracy of the ion gauges and the uncertainty in the estimate of the beamline volume. Uncertainty in this throughput measurement is $\pm 20 \%$. Second, the equivalent throughput associated with the extracted ions can be computed and compared to the pumpout (beamline pressure decrease) during beam extraction. 2 The equivalent throughput is computed from the beam current and the extracted $\mathrm{D}^{+}, \mathrm{D}_{2}{ }^{+}$, and $\mathrm{D}_{3}{ }^{+}$ratios. Gas throughput into the system, which is proportional to the beamline pressure before the beam was extracted, can then be deduced. The accuracy of this technique depends upon the accuracy to which the beam composition is known and the accuracy of measuring pressure ratios. Since the uncertainty of OMA diagnostic is $-5 \%$ (e.g., a $\mathrm{D}_{2}{ }^{+}$fraction of $0.20 \pm 0.01$ ) and pressure ratios can be estimated to $-10 \%$, the uncertainty of this throughput measurement is approximately $\pm 10 \%$. 


\section{RESULTS}

\section{A. Source operation}

It was intended that the experiment be performed at constant perveance even though the pressure in the plasma source was dramatically varied. It was further hoped that the chosen perveance would be close to the optimum perveance, namely $1.7 \times 10^{-6} \mathrm{~A} / \mathrm{V}^{1.5}(1.7 \mu$ perv). At the $95 \mathrm{kV}$ voltage at which the experiment was performed, optimum perveance corresponds to $50 \mathrm{~A}$ of extracted current. Initial operation resulted in unreliable operation due to grid faulting as the throughput was reduced. As a result, the experiment was carried out at a reduced perveance of $1.65 \mu$ perv ( $49 \mathrm{~A}$ at $95 \mathrm{kV}$ ). At the lowest gas settings, the perveance had to be reduced considerably below $1.65 \mu$ perv for reliable beam extraction. Also, at the lowest flow, noise on the Langmuir probes monitoring ion saturation current caused arc faults, resulting in arc termination. Arc fault protection is provided to prevent source operation in the low arc efficiency mode ${ }^{13}$ which is deleterious to the plasma generator. Detection of this condition results in termination of the are and any high voltage. As the gas throughput was reduced further, for either the source plus neutralizer or OMA gas feed, the arc would not strike at all.

For the source plus neutralizer gas feed, the neutralizer throughput was fixed at 15 torr-1/s. Gas throughput into the plasma source was varied from 3.9 torr-1/s to 1.6 torr $-1 / \mathrm{s} ; 3.9$ tors $-1 / \mathrm{s}$ being the nominal operating point. At 1.6 torr $-1 / \mathrm{s}$ the perveance had to be decreased to $1.54 \mu$ perv for the source to run; this value is to be compared to $1.65 \mu$ perv at 2.3 torr- $-1 / \mathrm{s}$, the next higher throughput in the sequence. 
With the OMA gas feed, the range of throughput variation was greater, 20 torr-1/s to 10.5 torr-1/s. The source would operate here at throughputs well below that for the source plus neutralizer case. At 10.5 torr- $1 / \mathrm{s}$, the highest perveance at which the source would operate was $1.46 \mu$ perv.

\section{B. Accelerator grid currents}

During beam extraction, currents to three of the four grids are monitored. Since the source grid is at the full accelerating potential, the current from this grid equals the extracted ion current. Currents to the middle two grids, the gradient and suppressor grids are of more interest. These were found to be strong functions of the gas throughput. Currents to these grids are due to the collection of ions or electrons, or the emission of secondary particles due to the interception of charged or neutral particles. Charge exchange of accelerating ions with gas in the accelerator is of primary concern. Charge exchange creates boch aberrant ion and neutral particles that can strike the grids. It reduces the useful beam power by creating a spectrum of low energy particles.

Figures 2 and 3 show the behavior of the gradient and suppressor grid currents, respectively, as a function of total gas throughput for both gas injection configurations. Solid circles are for the OMA gas feed and open circles are for the source plus neutralizer gas feed; this same designation is maintained on all succeeding figures. Suppressor currents are higher because it is farther downstream and better able to intercept aberrant particles, it is preceded by a long path length for charge exchange, and it is bombarded by ions from the neutralizer plasma. The suppressor current was found to be linear with throughput for both 
gas injection cases, implying that charge exchange is still occurring at the lowest throughputs examined. By comparison, the gradient grid current is non-linear, approaching an asymptotic value at low throughput.

Currents are larger, at the same throughput, for the OMA gas feed. At the lowest throughputs at which the source would operate, the suppressor currents were equivalent for the two cases, whereas the gradient grid current was higher (although still low) for the OMA case.

\section{Beam composition}

The fractions of ions accelerated as $\mathrm{D}^{+}, \mathrm{D}_{2}{ }^{+}$, and $\mathrm{D}_{3}{ }^{+}$were measured with the OMA. Beam composition as a function of throughput for the two injection configurations are shown in figure 4. These fractions are those of the three ion constituents $\left(\mathrm{D}^{+}, \mathrm{D}_{2}{ }^{+}\right.$, and $\mathrm{D}_{3}{ }^{+}$) projected back to the accelerator; i.e., they represent ion fractions at the exit plane of the accelerator. ${ }^{16}$

(Beam composition measured with the original gas feed system, i.e., into the ion source only, was within measurement tolerance of those measured here at high throughput.)

Gas throughput, and how the gas is injected, has an effect on the beam composition. At high throughput, results for the two cases are the same; at the lowest throughputs, they differ. The $\mathrm{D}^{+}$fraction is $66 \%$ at 10.5 torr- $1 / \mathrm{s}$, the lowest throughput for the OMA case, versus $72 \%$ at 16.6 torr-1/s, the lowest throughput for the source plus neutralizer case. In both cases, the $\mathrm{D}^{+}$and $\mathrm{D}_{3}{ }^{+}$fractions are directly proportional to the throughput whereas the $\mathrm{D}_{2}{ }^{+}$fraction is inversely proportinnal. 


\section{Power}

Figure 5 shows the behavior of the neutral power delivered to the calorimeter as a function of throughput. Power is normalized by the extracted ion current to correct for small perveance changes in the data. Given the suppressed zero of the ordinate, power did not vary very much. The lowest throughput data point is not shown since its perveance is considerably different than the rest. Power for this point lies well below the data shown. Since its perveance and hence divergence are different from the rest of the data, it is uncertain how much of the power reduction is due to poor beam optics and how much to under-neutralization. So as to compare data with similar beam optics, this point is omitted. The scatter of dat $\hat{a}$ in figure 5 is at the level of the estimated $\pm 5 \%$ random error of the power measurement. ${ }^{19}$ Therefore, the observed variation in power with throughput is not deemed significant. Thermocouples embedded in the full-energy ion dump also exhibited minimal variation with throughput.

\section{DISCUSSION}

\section{A. Source operation}

Pressures in the ion source can be estimated from the gas flow rates and conductances of the neutralizer and accelerator grids. ${ }^{20}$ Room temperature molecular flow is assumed. Neutralizer conductances are calculated to be $3000 \mathrm{l} / \mathrm{s}$ from the OILA feed point to the beamline, $6000 \mathrm{l} / \mathrm{s}$ from the neutralizer gas feed point to either the beamline or the accelerator, and $3000 \mathrm{l}$ 's from the OMA through the accelerator grid structure into the plasma generator. ${ }^{21}$ Errors in computing the relative conductances, especially the accelerator grid conductance, are $\pm 20 \%$. 
For the source plus neutralizer gas feed configuration, a superposition of the two pressure distributions was used. When calculating pressures for either the OMA or the neutralizer, it was assumed that the pressure was constant from the feed point to the ion source. This is a good assumption for computing pressure distributions during the arc phase of a beam pulse, but is in some error when beam is extracted, since gas flow from the neutralizer to the source (to make the beam) requires a pressure difference. While absolute pressures may be in error due to gas heating effects in the neutralizer, ${ }^{22}$ comparison of relative pressures and flow rates between the two gas injection configurations is possible.

Source pressure was calculated for the lowest throughputs at which the source could sustain an arc. Both cases, -10 torr-1/s into the OMA and a combination of 15 torr-1/s into the neutralizer and 1.5 torr-1/s into the ion source, yield a source pressure of $-3.5 \times 10^{-3}$ torr. Agreement between the two systems was excellent, i.e., within $6 \%$. For pressures below this value, there is insufficient gas to sustain a discharge. At slightly higher pressures, beam operation was only possible at reduced perveance. Under these gas starved conditions the gas density can only support a low plasma density regardless of arc power. Plasma density is also affected by the change in pressure when the beam is extracted.

\section{B. Gas efficiency}

At the extracted beam current of $50 \mathrm{~A}$ for this experiment, and for the measixred beam composition of $75 \% \mathrm{D}^{+}, 15 \% \mathrm{D}_{2}{ }^{+}$, and $10 \% \mathrm{D}_{3}{ }^{+}$, the gas flow rate necessary to provide the beam particles is 5.8 torr-1/s. For the OMA gas feed, and the given conductances, at most (for zero source pressure) 50\% of the gas entering the neutralizer flows to the ion scurce. Half of the gas flows each direction since the conductances to the source and into the beamline are equal. The lowest 
throughput at which the source would operate was abcut 10 torr-l's. 'This value, taken together with the combined lincertainties of the throughput measurements and the conductance calculations, implias that the gas reaching the ion source is converted into beam ions with greater than $75 \%$ efficiency. The degree of ionization of the piasma in ine ion source is probably significantly less than $75 \%$. It can be estimated by equating the flux of ions leaving the source with that of the neutrals entering; it is given by the ratio of the neutral particle velocity to that of the ion drifting to the extraction sheath. Using soom temperature for the neutrals and $1 \mathrm{eV}$ for the ions, ${ }^{23}$ a fractional ionization of $-15 \%$ is calculated.

For the source plus neutralirer gas feed syrstem, the minimum gas flow condition wfs 1.5 torr-i/s of gas directly into the ion source and 15 torr-l/s into the neutralizer. The condurtance from the neutralizer feed point to the ion source is $2000 \mathrm{l} / \mathrm{s}(6000 \mathrm{l} / \mathrm{s}$ in series sith $3000 \mathrm{l} / \mathrm{s}$ ) versus $6000 \mathrm{~V} / \mathrm{s}$ to the beamline. Therefore, the maximum fraction of trip ceutralizer gas that can flow to she ion source is $25 \%$, or $3.8 \mathrm{tcrr}-1 / \mathrm{s}$. Gas efficiency of the plasma generator again is estimated to be greater than $75 \%$.

\section{Charge exchange in the accelerator}

Loss of ions in the accelerator through charge exchange can be calculated from the estimated grid pressure. Consider 20 torr $-1 / \mathrm{s}$ of gas flowing into the OMA port. Subtracting the 5.8 torr-1/s (beam particles) flowing into the ion source and dividing by the conductance to the beamline yields a ressure of $5 \times 10^{-3}$ torr at the ground grid. It has been shown, however, that the line density of the neutralizer is anomalously low if room temperature conductances are assumed: ${ }^{2,24}$ A model based upon heating of the neutralizer gas by the beam has beon proposed to explain these results. ${ }^{22}$ For TFTR, the reduction in the line 
density from room temperature conditions is three. ${ }^{2}$ Therefore, the accelerator pressure for the present calculation is reduced from $5 \times 10^{-3}$ torr to $1.6 \times 10^{-3}$ torr. For simplicity, this pressure is assumed throughout the entire grid structure. The particle energy was caiculated from the electric fields in the accelerator for a $95 \mathrm{kV}$ beam: $15 \mathrm{kV} / \mathrm{cm}$ over the $0.6 \mathrm{im}$ between the source and gradient grids, 63 $\mathrm{kV} / \mathrm{cm}$ for $1.3 \mathrm{~cm}$ between the gradieni and suppressor grids, and $-2 \mathrm{kV} / \mathrm{cm}$ over the $1 \mathrm{~cm}$ from the suppressor and ground giids. ${ }^{25}$ Given this field configuration, the energy of a $\mathrm{D}^{+}$ion was computed as a function of location within the accelerator. Electron capture cross sections were taken from reference 25.

Under these conditions, the charge exchange losses in figure 6 result. Parcicle energy and loss were computed using a $1.5 \mathrm{~mm}$ step size. Both the incremental loss (open circles) and the accumulated loss (solid circles) are shown. Note that the loss per unit length is greatest near the source grid where the particle velocity is lowest; since the electron capture cross section peaks for energies between 4 and $20 \mathrm{keV}$. For this high throughput case, $-7 \%$ of the $\mathrm{D}^{+}$extracted from the plasma generator is predicted to charge exchange in the accelerator. Similar losses would be expected from the minority components, $\mathrm{D}_{2}{ }^{+}$and $\mathrm{D}_{3}{ }^{+}$. At the lowest OMA gas feed, namely $\sim 10 \mathrm{tcm}-1 / \mathrm{s}$, the accelerator pressures, pind henc : charge exchange loss, are calculated to be 3.5 times less, or approximately 29 . This predicted ratio of 3.5 is approximately what was observed in the gradient grid current as the throughput was reduced from 20 torr-1/s to 10 tor $-1 / \mathrm{s}$.

We can speculate further that when the grid currents for the two gas feed systems are equal that the accelerator pressures would be equal. Based on figures 2 and 3, this would imply that 19 torr.l/s of gas into the source plus neutralizer 
and $\sim 13$ torr-1/s into the OMA should have equal accelerator pressures. Gas flow calculations based upon our simple model predict that the accelerator pressures for the two cases are the same within $5 \%$.

\section{Power}

In 1989 we reported that the neutral fraction of the beam was a function of gas throughput into the ion source. ${ }^{2}$ Operation at that time was with gas fed only into the back of the plasma generator. Figure 7, taken from this earlier study, shows the neutral fraction of the beam as a function of neutralizer line density as the throughput was varied from 13 to 21 torr-Vs. Neutral fractions for the data points connected by the dotted line were measured on the beamline calorimeter on succeeding pulses with the magnet on and then off. Neutralizer line densities for these points were computed from the gas throughput assuming room temperature molecular flow. The solid line is the cásulated neutral fraction versus line density. The difference between the data points and the prediction has been attributed to elevated gas temperature. This figure clearly shows the neutral fraction falling with decreasing throughput as gas is consumed to make the beam (leaving less to provide a neutralizing medium).

In the present experiment, it is believed that charge exchange in the grids is competing with beam neutralization. Adding gas to the system improves the reutral fraction of the beam (not measured in the present experiment) while causing increased charge exchange loss during the acceleration process. A second order effect is the change in beam composition as a function of throughput. Since the neutralization fractions for incident $\mathrm{D}^{+}, \mathrm{D}_{2}{ }^{+}$, and $\mathrm{D}_{3}{ }^{+}$are different, changes in the beam $c c$ nposition also affect the neutral beam power. 
The net result of these competing effects is a neutral beam power relatively constant over the range of throughputs examined. Further optimization studies are planned to explore this competitive phenomenon.

\section{E. Future operation}

During the forthcoming TFTR operational period we will contmue tc operate ion sources with gas fed into the OMA to gain experience and confidence pertinent to future tritium gas injection. A nominal throughput of 13 to 14 torr-1/s is a reasonable compromise between the competing neutralization and accelerator charge exchange processes, as well as providing an operating point away from arc and beam faulting problems. Charge exchange losses in the accelerator will be equivalent to those experienced during previous long pulse ion source operation.

An ideal gas system, which unfortunately cannot be realized on the existing TFTR beamlines, would be to decouple the : 3 utralizer from the ion source. The problems of gas pressure in the accelerator. A neutralizer line density would then be independent and could be independer. $r$ optimized. This is especially attractive for the condition of high gas efficiency, which has been argued to exist on TFTR long pulse ion source. Future neutral beam systems could utilize such a neutralization scheme.

\section{F. Summary}

It has been demonstrated that TFTR ion sources can be operated with gas injected into the neutralizer at a location close to the accelerator. The use of such a system with tritium will greatly simplify the plumbing and control electronics. 
In addition, it has been found that less gas is required with such a system than when gas $i s$ added to the neutralizer plus the ion source. This reduction is $36 \%$, from 19 torr-l/s to 14 torr-l/s, a significant potential savings in tritium consumption. The injected power is unchanged, $-2.2 \mathrm{MW}$ at $95 \mathrm{kV}$. The $\mathrm{D}^{+}$ fraction of the beam is reduced from $77 \%$ for the source plus neutralizer gis system to $71 \%$ for the new operating point. This change is slightly greater than the $\pm 5 \%$ absolute accuracy (due to cross section and neutralizer line density uncertainties) of the OMA diagnostic, but is significantly greater than the random error. At the same time, the $D_{2}{ }^{+}$fraction of the beam increased from 18 to $26 \%$.

\section{ACKNOWL EDGMENTS}

We wish tc acknowledge the assistance of Vic Garzotto, John Desandro, and Fred Simmands, Ir. for the mechanical installation of the gas injection systems and Mark Cropper and Doug Westover for interlocks and instrumentation. The assistance of Larry Lagin and George Kolinchak, of the computer division, is gratefuily acknowledged for maintenance of the software and hardware, respectively. We further wish to thank and neutral beam operations team for their capable operation of the ion sources and the cryogenic system. This work is supported by the U. S. Department of Energy under contract No. DE-ACO276 บHO3073. 


\section{REFERENCES}

1J. D. Strachan, M. Bitter, A. T. Ramsey, M. C. Zarnstorf, et al., Phys. Rev. Lett. 58, 1004 (1987)

2J. H. Kamperschroer, G. M. Gammel, H. W. Kugel, L. R. Grisham, T. N. Etevenson, A. on Haile, M. D. Williams, and T. T. C. Jones, J. Vac. Sci. Technol. A 7, 83 (1989).

${ }^{3}$ L. R. Grisham, H. F. Dylla, M. D. Williams, K. Wright, and R. Causey, J. Vac. Sci. 'Technol. A 7, 944 (1989).

${ }^{4}$ L. R. Grisham, H. D. Falter, R. Hemsworth, and G. Deschamps, Rev, Sc: Instrum. 60, 3730 (1989).

${ }^{5}$ L. R. Grisham, T. Stevenson, K. Wright, H. Falter, R. Causey, and W. Christman, accepted for publication in Rev. Sci. Instrum.

${ }^{6} \mathrm{H}$. D. Falter, private communication.

${ }^{7}$ L. C. Pittenger, in Proceedings of the Sixth Symposium on Engineering Problems of Fusion Research, San Diego, 1975 (IEFE, New York, 1976), p. 700.

8t. C. Pittenger, R. R. Stone, L. E. Valby, and L. R. Pedrotti, in Proceedings of the Seventh Symposium on Engineering Problems of Fusion Research, Knoxville, 1977 (IEEE, New York, 1977), Vol. 1, p. 555.

${ }^{9}$ M. D. Williams, H. P. Eubank, L. R. Grisham, J. H. Kamperschroer, H. W. Kugel, G. D. Martin, T. O'Connor, R. E. Prechter, B. A. Prichard, Jr., A. von Halle, R. A. Winje, and K. E. Wright, Fusion Technol. 8, 800 (1985).

${ }^{20}$ T. Stevenson, G. M. Gammel, L. R. Grisham, J. Kamperschroer, A. von Halle, and M. D. Williams, in Proceedings of the Twelfth Symposium on Fusion Engineering, Monterey, 1987 (IEEE, New York, 1987), Vol. 2, p. 1141. 
${ }^{11}$ T. Stevenson, A. von Halle, L. Dudek, L. Grisham, T. O'Connor, and M. Williams, in Proceedings of the Thirteenth Symposium on Fusion Engineering, Knoxville, 1989 (IEEE, New York, 1990), Vol. 1, p. 292.

${ }^{12}$ M. C. Vella, W. S. Cooper, P. A. Pincosy, R. V. Pyle, P. D. Weber, and R. P. Wells, Rev. Sci. Instrum. 59, 2357 (1988).

${ }^{13}$ P. A. Pincosy, K. W. Ehlers, A. F. Lietzke, H. M. Owren, J. A. Paterson, R. V. Pyle, and M. C. Vella, Rev. Sci. Instrum. 57, 2705 (1986).

${ }^{14}$ P. D. Weber, H. M. Owren, J. A. Paterson, P. A. Pincosy, R. V. Pyle, R. P. Wells, and M. C. Vella, Rev. Sci. Instrum. 57, 2714 (1986).

${ }^{15}$ L. E. Valby, in Proceedings of the Seventh Symposium on Engineering Problems of Fusion Research, Knosville, 1977 (IEEE, New York, 1977), Vol, 2, p. 1040 .

${ }^{16}$ J. H. Kamperschroer, H. W. Kugel, M. A. Reale, S. L. Hayes, G. A. Johnson, J. L. Lowrance, P. A. Shah, P. Sichta, B. W. Sleaford, M. D. Williams, and P. M. Zucchino, Rev. Sci. Instrum. 58, 1362 (1987).

${ }^{17}$ Veeco Instruments, Vacuum Products and Instrumentation Division, Plainview, NY 11803.

${ }^{18}$ Maxtek, Inc., Torrance, CA 90503.

19 J. H. Kamperschroer, L. R. Grisham, L. E. Dudek, G. M. Gammel, G. A. Johnson, H. W. Kugel, L. Lagin, T. E. O'Connor, P. A. Shah, P. Sichta, T. N. Stevenson, A. von Halle, M. D. Williams, and R. Bastasz, Rev. Sci. Instrum. 60, 3377 (1989).

${ }^{20}$ S. Dushman, Scientific Foundations of Vacuum Technique, Second Edition, edited by J. M. Lafferty (John Wiley and Sons, New York, 1962). 
${ }^{21}$ Neutralizer conductances were computed from reference 20 , accelerator grid conductances are taken from J. E. Willis and K. H. Berkner, in Proceedings of the Ninth Symposium on Engineering Problems of Fusion Research, Chicago, 1981 (IEEE, New York, 1981), Vol. 1, p. 798.

${ }^{22}$ J. Pamela, Rev. Sci. Instrum. 57, 1066 (1986).

${ }^{23}$ C. F. Chan, C. F. Burreli, and W. S. Cooper, J. Appl. Fhys. 54, 6119 (1983).

${ }^{24}$ H. D. Falter, R. S, Hemsworth, G. H. Deschamps, A. P. H. Goede, T. T. C. Jones, P. Massmann, M. J. Mead, and A. Stabler, in Proceedings of the Eleventh Symposium on Fusion Engineering, Austin, 1985 (IEEE, New York, 1986), Vol. 2, p. 786.

${ }^{25}$ LBL/LLC CTR Staff, Lawrence Berkeley Laboratory Report No. LBL-3296, 1975.

${ }^{26}$ C. F. Barnett, J. A. Ray, E. Ricci, M. I. Wilker, E. W. McDaniel, E. W. Thomas, and H. B. Gilbody, Ouk Ridge National Laboratory Report No. ORNL-5206 (vol. 1), 1977. 


\section{FIGURE CAPTIONS}

Figure 1. Schematic of the three TFTR neutral beamline gas feed points. From left to right they are: back of the plasma generator, through the OMA chamber into the neutralizer, and the neutralizer midpoint.

Figure 2. Gradient grid current as a function of total gas throughput for gas fed into the ion source plus neutralizer (open circles), and into the OMA location (solid circles).

Figure 3. Suppress:r grid current as a function of total gas throughput for gas fed into the ion source pins neutralizer (open circles), and into the OMA location (solid circles).

Figure 4. Beam composition as a function of total gas throughput. The measurement is from the Doppler-shifted emission of the beam, and is computed as fractions of the beam extracted from the source as $\mathrm{D}^{+}$, $\mathrm{D}_{2}{ }^{+}$, and $\mathrm{D}_{3}{ }^{+}$. Open circles are for the source plus neutralizer gas feed and the solid circles are for the OMA gas feed.

Figure 5. Neutral power measured on the calorimeter, normalized to the extracted beam current, versus the total gas throughput. Solid circles are for the OMA gas feed and the open circle is for the source plus neutralizer gas feed. 
Figure 6. Calculated charge exchange lose of $\mathrm{D}^{+}$in the accelerator for an OMA gas throughput of 20 torr-1/s. The abscissa designates position within the accelerator, zero heing at the source grid. Open circles are the incremental loss per $1.5 \mathrm{~mm}$ step, and solid circies tid the accumulated loss.

Figure 7. Neutral fraction measured calorimetrically on the beamline calurimeter varsus the calculated neutralizer line density (data points ccanected with the dotted ine). The solid line is the predicted neutral fraction for a beam composition similar to that measured during this experiment. 
PPPL $\$ 91 \times 0067$

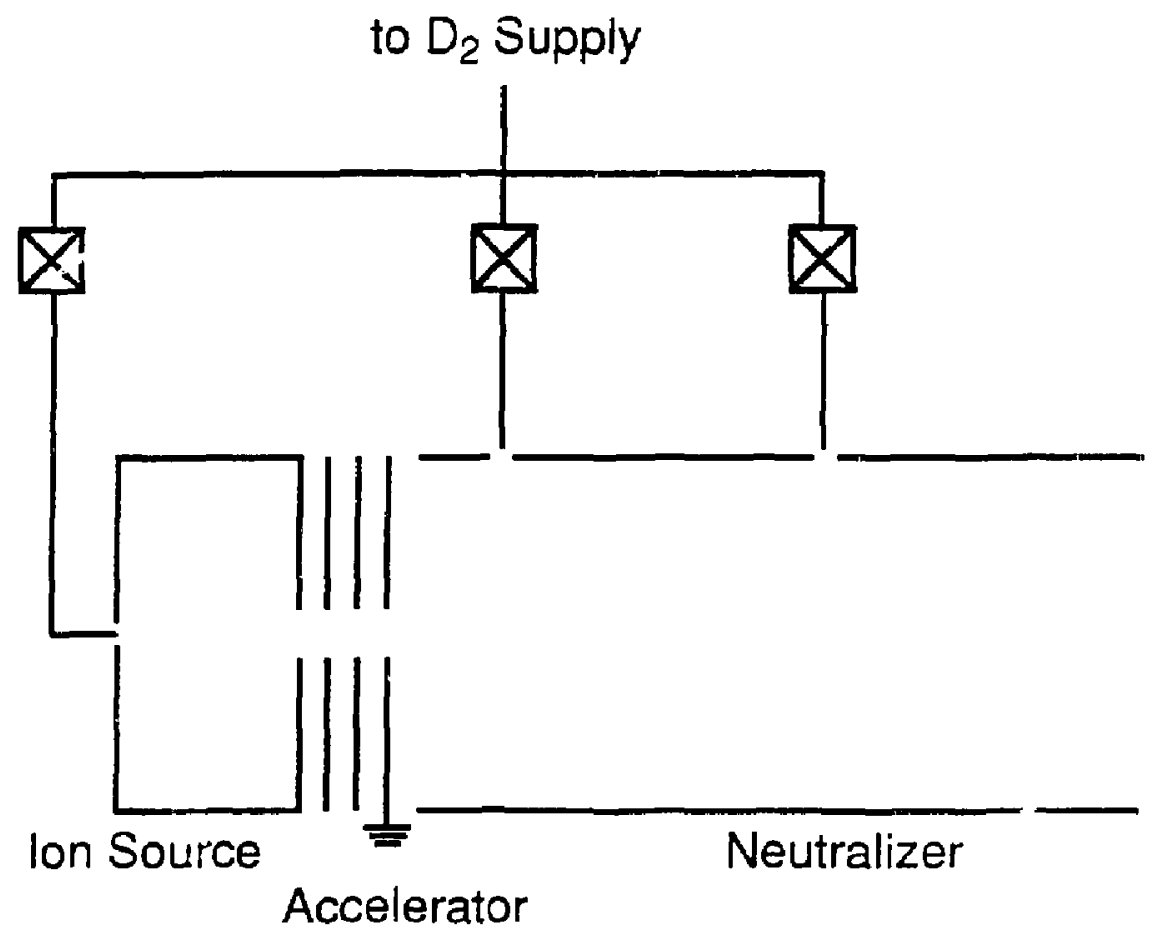

FIG, 1 


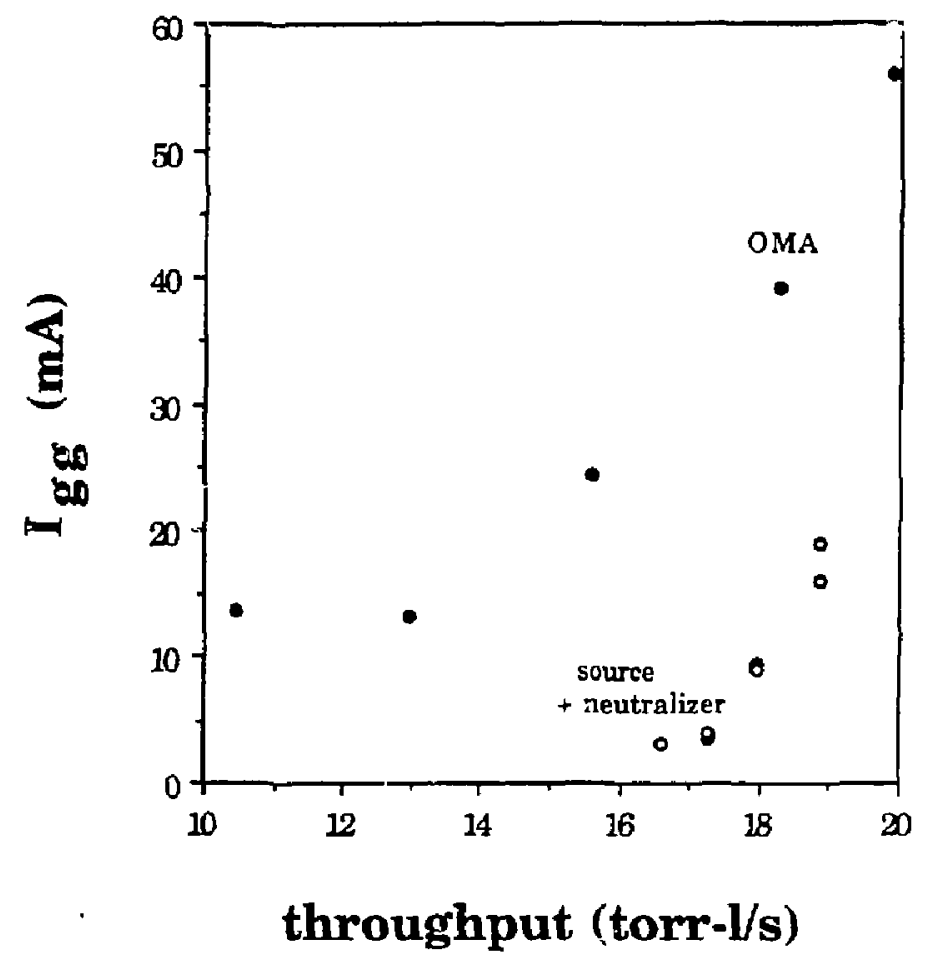

FIG. 2 


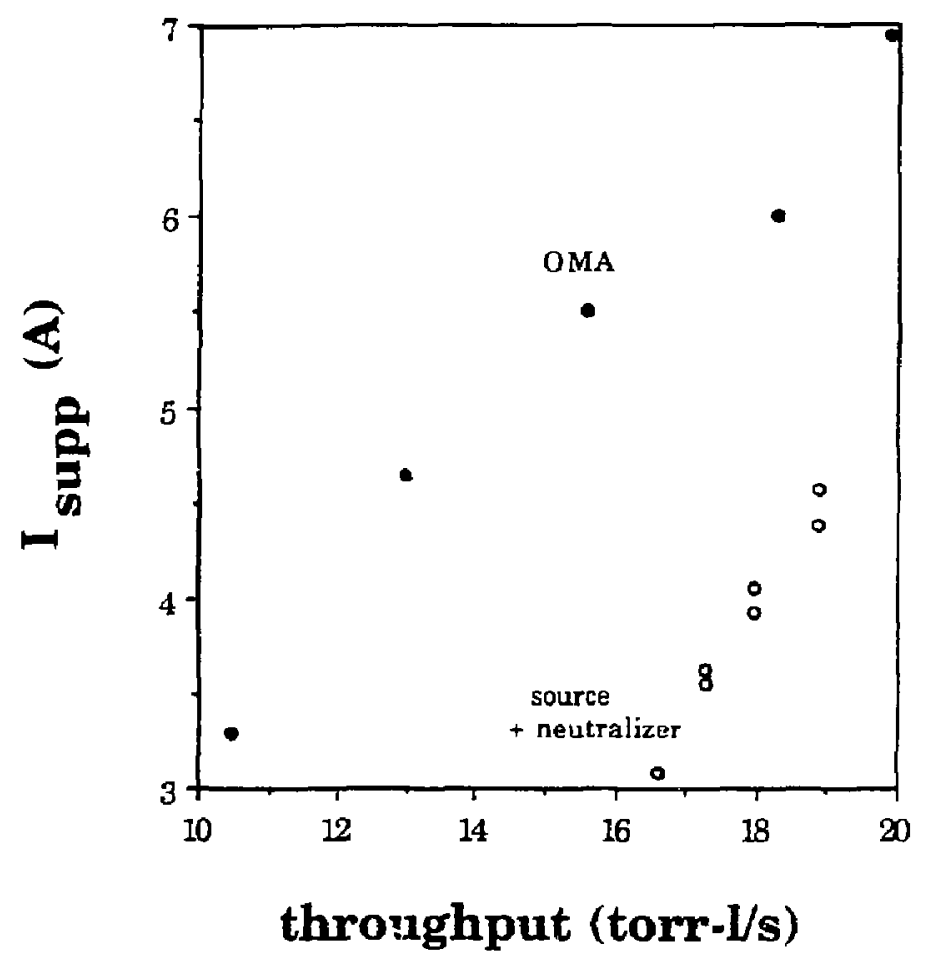

FIG, 3 
beam composition (\%)

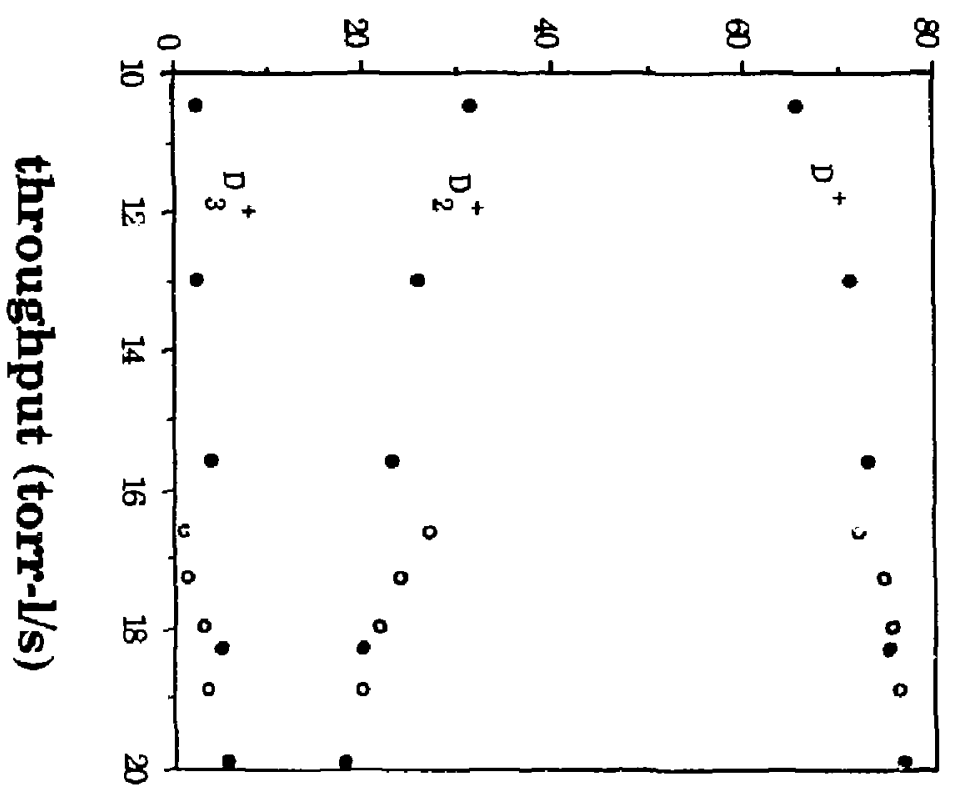




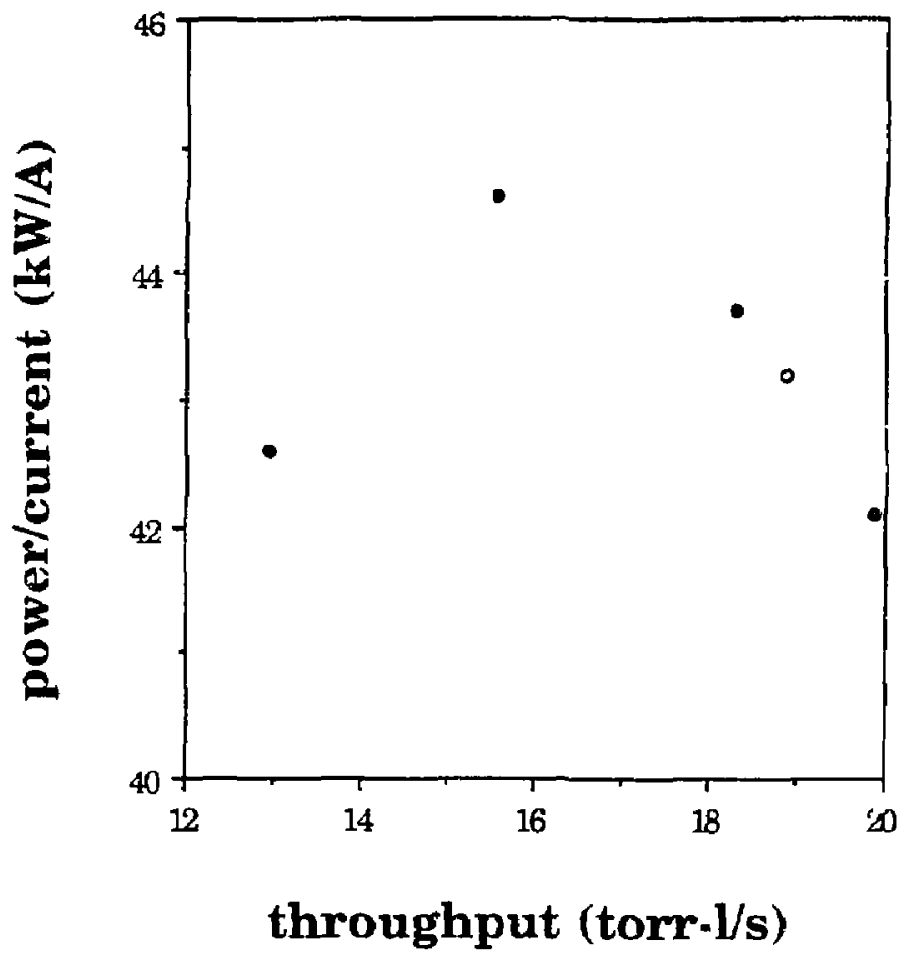

FIG. 5 


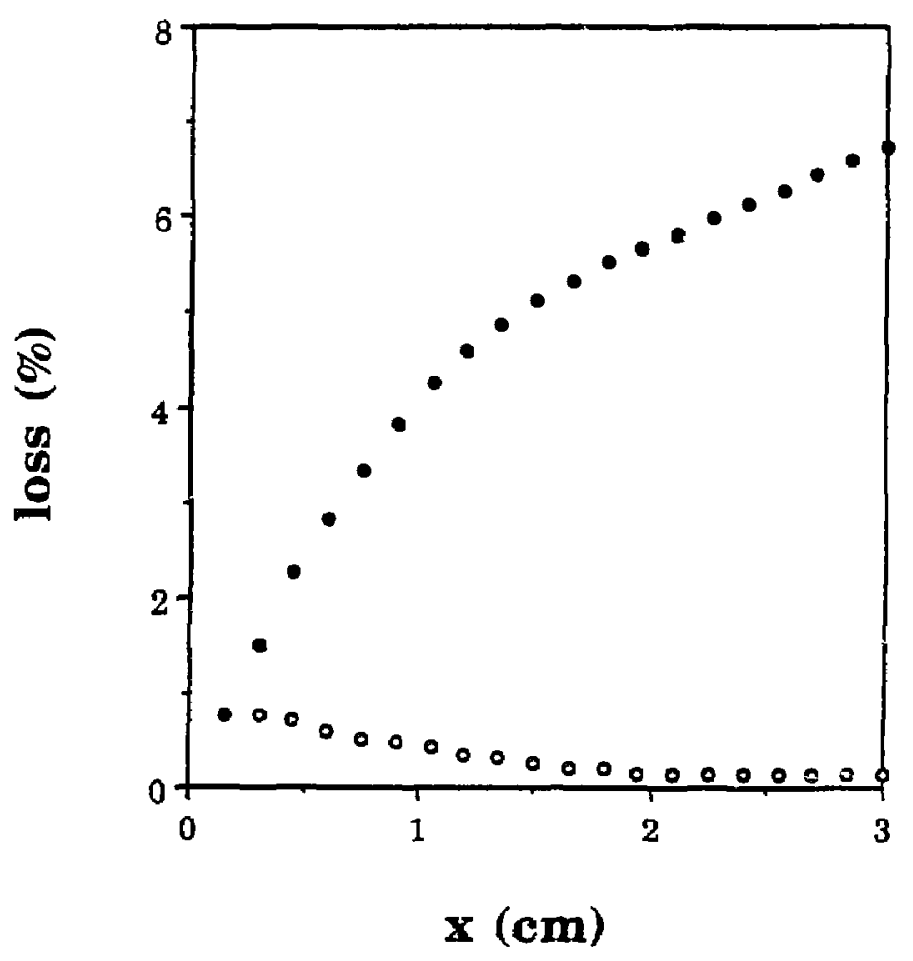

FIG, 6 


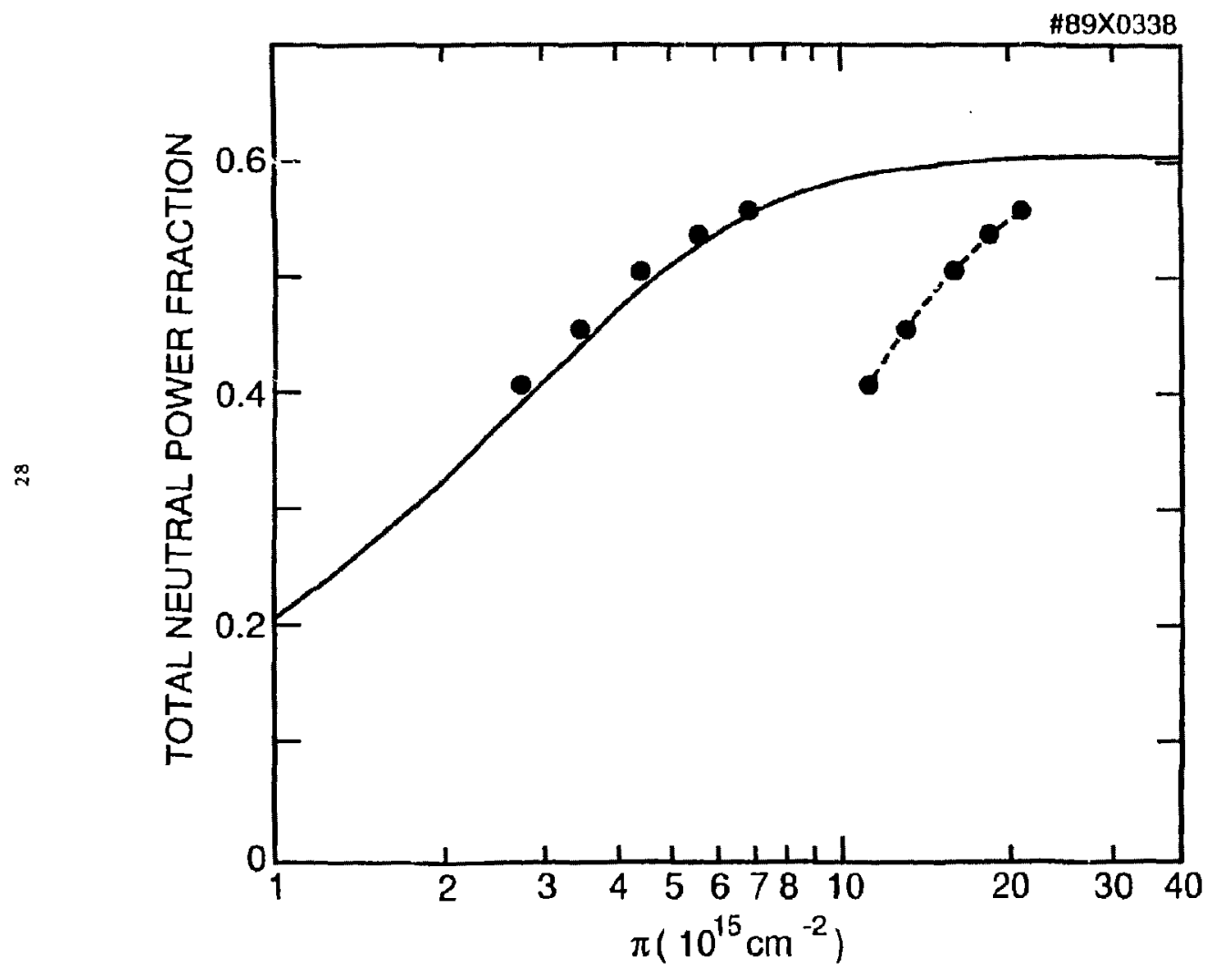

号 